\title{
The role of biological system other than auditory air-conduction in the emergence of the hypersonic effect
}

Tsutomu Oohashi ${ }^{1,2}$, Norie Kawai ${ }^{1}$, Emi Nishina ${ }^{3,4}$, Manabu Honda ${ }^{5,6}$, Reiko Yagi ${ }^{1,5}$, Satoshi Nakamura ${ }^{6}$, Masako Morimoto ${ }^{5,7}$, Tadao Maekawa ${ }^{8}$, Yoshiharu Yonekura ${ }^{9,10}$, Hiroshi Shibasaki ${ }^{11,12}$

${ }^{1}$ Department of Research and Development, Foundation for Advancement of International Science, Tokyo 164-0003 Japan

${ }^{2}$ National Institute of Information and Communications Technology, Koganei 184-8795 Japan

${ }^{3}$ Research and Development Department, National Institute of Multimedia Education, Chiba 261-0014 Japan

${ }^{4}$ School of Cultural and Social Studies, The Graduate University for Advanced Studies, Kanagawa 240-0193 Japan

${ }^{5}$ Department of Cortical Function Disorders, National Institute of Neuroscience, National Center of Neurology and Psychiatry, Kodaira 187-8502 Japan

${ }^{6}$ SORST, Japan Science and Technology Agency, Kawaguchi 332-0012 Japan

${ }^{7}$ Faculty of Social Information, Jumonji University, Niiza 352-8510 Japan

${ }^{8}$ Faculty of Environmental and Information Sciences, Yokkaichi University, Yokkaichi 512-8512 Japan

${ }^{9}$ Biomedical Imaging Research Center, University of Fukui, Fukui 910-1193 Japan

${ }^{10}$ National Institute of Radiological Sciences, Chiba 263-8555 Japan

${ }^{11}$ Human Brain Research Center, Kyoto University Graduate School of Medicine, Kyoto 606-8507 Japan

${ }^{12}$ National Institute of Neurological Disorders and Stroke, NIH, Bethesda, MD 20892 USA

Number of text pages: 26 pages

Number of figures and tables: 3 figures and no table

Corresponding author:

Tsutomu Oohashi, Ph.D.

Foundation for Advancement of International Science 1-53-11 Higashinakano, Nakano-ku, Tokyo 164-0003, Japan

Tel. +81-3-3366-8740 Fax. +81-3-3366-8737

e-mail: oohashi@fais.or.jp

For Systems Neuroscience Section, Brain Research (2nd revision) December 16, 2005 


\begin{abstract}
Although human beings cannot perceive elastic vibrations in the frequency range above $20 \mathrm{kHz}$, non-stationary sounds containing a wealth of inaudible high-frequency components (HFC) above the human audible range activate deep-lying brain structures, including the brainstem and thalamus and evoke various physiological, psychological, and behavioral responses. In the previous reports we have called these phenomena collectively "the hypersonic effect." It remains unclear, however, if vibratory stimuli above the audible range are transduced and perceived solely via the conventional air-conducting auditory system or if other mechanisms also contribute to mediate transduction and perception. In the present study, we have examined the emergence of the hypersonic effect when inaudible HFC and audible low-frequency components (LFC) were presented selectively to the ears, an air-conducting auditory system, or to the body surface including the head which might contain some unknown vibratory sensing mechanisms. We used two independent measurements based on differing principles; one physiological (alpha 2 frequency of spontaneous electroencephalogram [alpha-EEG]) and the other behavioral (the comfortable listening level [CLL]). Only when the listener's entire body surface was exposed to HFC, but not when HFC was presented exclusively to the air-conducting auditory system, did both the alpha-EEG and the CLL significantly increase compared to the presentation of LFC alone, that is to say, there was an evident emergence of the hypersonic effect. The present findings suggest that the conventional air-conducting auditory system alone does not bring about the hypersonic effect. We may need to consider the possible involvement of a biological system distinct from the conventional air-conducting auditory nervous system in sensing and
\end{abstract}


transducing high-frequency elastic vibration above the human audible range.

Classification terms:

Theme: Sensory systems $\quad$ Topics: Auditory systems: central physiology

Section: Systems Neuroscience, Sensory and Motor Systems

Key word: Auditory; Non-auditory; Vibration; Electroencephalogram; Comfortable

listening level; Body surface 


\section{Introduction}

It is generally accepted that human beings cannot perceive elastic vibrations in the frequency range above $20 \mathrm{kHz}$ as sound. Nevertheless, a non-stationary sound containing significant quantities of fluctuating high-frequency components (HFC) beyond the human audible range evokes a significant increase in the regional cerebral blood flow $(\mathrm{rCBF})$ in the brainstem and thalamus and in the occipital alpha frequency component of the spontaneous electroencephalogram (EEG) as compared with an otherwise identical sound from which the HFC are removed (Oohashi et al., 1991, Oohashi et al., 2000, Oohashi et al., 2001, Yagi et al., 2002, Yagi et al., 2003a). In addition, the inclusion of HFC makes a sound more pleasant to hear and evokes a specific behavior, that is, the listeners spontaneously adjust the comfortable listening level (CLL) of the sound to a greater magnitude (Oohashi et al., 1991, Oohashi et al., 2001, Yagi et al., 2002, Yagi et al., 2003a, Yagi et al., 2003b). We call such phenomena collectively "the hypersonic effect." The discovery of this effect (Oohashi et al., 1991) has strongly impacted the audio industry; cutting edge digital audio media, such as super audio compact disc (SACD) and digital versatile disc audio (DVD-Audio), allow the recording of inaudible HFC. The biological mechanisms underlying the emergence of the hypersonic effect, however, remain unknown.

Although the hypersonic effect is a set of phenomena closely related to the human auditory function, it contains some unusual characteristics. In humans, for example, it is known that air vibration frequencies above $20 \mathrm{kHz}$ are poorly conducted to the inner ear for several reasons, such as the mechanical property of the auditory ossicles in the middle ear and the basilar membrane in the inner ear. Moreover, none of the 
physiological, psychological or behavioral responses of the hypersonic effect is ever observed by HFC alone. This suggests that the biological effect of HFC likely appears only as a result of some complex interaction between HFC and audible low-frequency components (LFC) rather than as a simple response to HFC. We cannot give a consistent explanation of the hypersonic effect's biological mechanism by conventional auditory physiology.

In this study, as the first step in exploring this biological mechanism, we conducted tests to determine if these unique phenomena are a simple solitary response of the auditory nervous system mediating air-conduction, and furthermore if we can eliminate the involvement of a non-air-conducting auditory system or any other biological system from the contributing factors. For this purpose, we have divided the sound source, which has been proven to induce the hypersonic effect (Oohashi et al., 2000), into two components: an audible LFC and an inaudible HFC. While the LFC was presented to the air-conducting auditory system, the HFC was simultaneously presented either to the air-conducting auditory system or to the entire body surface; in the latter various vibratory sensing systems other than the air-conducting auditory system might exist. Under each condition, we compared the full-range sound (FRS: simultaneous presentation of LFC and HFC) and LFC alone. In this way we examined if there were any differences in the emergence of the hypersonic effect under these different conditions.

To investigate the possible participation of some unknown vibration sensing systems in some other parts of the human body excluding the ears, the entire body surface of a subject should, ideally, be exposed to the HFC. At the same time, elastic vibratory 
noise other than the experimental sound materials should be adequately eliminated from the experimental setup. Functional magnetic resonance imaging (MRI) and positron emission tomography (PET), which are at present widely used for noninvasive study of brain functions, are not suitable for the purpose of the present study, namely, to examine the possible participation of some unknown vibration-sensing system other than air-conducting auditory nervous system in the hypersonic effect. The borehole structure of MRI and PET scanners, inside which subjects must remain in a prone position during image acquisition, prevents experimental sounds, especially the HFC, from reaching the entire body surface of the subjects. Moreover, an MRI scanner, with its rapid change of magnetic gradient, produces a strong mechanical vibration resulting in an extremely loud noise that masks the experimental sound, which is a critical drawback. PET scanners also emit a mechanical vibratory noise, including fan noise, transmitted through the air, and its scanner bed often contains a considerable quantity of frequency component above the human audible range that contaminates the experimental sound and makes it unclear whether the experimental sound contains HFC or not. These vibratory noises are extremely difficult to eliminate. Our pilot study using functional MRI and PET failed to show a clear contrast between different experimental conditions. Thus, we have found MRI and PET of ordinary use inappropriate for this research.

In the present study, therefore, we selected two differing measurements that are free from the above limitations and were clearly able to detect the emergence of the hypersonic effect in the previous studies: (1) physiological measurement of spontaneous EEG using a portable multi-channel telemetry system and (2) behavioral measurements on CLL (Yagi et al., 2003a, Yagi et al., 2003b, Oohashi et al., 2001, Yagi et al., 2002). 
These measurement are based on differing principles and independent of each other. Measuring the spontaneous EEG with the portable multi-channel telemetry system is advantageous because this system does not produce a mechanical vibration and does not require subjects to lie down during data acquisition, thus enabling sounds to easily reach the entire body surface of the subjects in seated or standing positions. As an electrophysiological index of the hypersonic effect, we used the averaged power of the alpha 2 frequency range $(10-13 \mathrm{~Hz})$ of spontaneous EEG (alpha-EEG) recorded from the centro-parieto-occipital region. This index parallels the rCBF change in the deep-lying brain structure induced by sounds containing HFC (Nakamura et al., 2004).

Converging results of the EEG and CLL measurements both made in the present study reveal that the hypersonic effect was not induced when the HFC was presented selectively to the air-conducting auditory system, but was induced when the HFC was presented to the entire body surface, including the head but excluding the ears. It seems difficult, therefore, to set up valid models for the human biological response to air vibration containing rich HFC above the audible range if we posit only the involvement of the conventional air-conducting auditory system by ignoring the possible involvement of some other biological system.

\section{Results}

EEG experiment. When both LFC and HFC (i.e., FRS) were presented through speakers to subjects ( 5 males and 7 females, $25-51$ years of age), the alpha-EEG power was significantly greater as compared with the presentation of the LFC alone, confirming the emergence of the hypersonic effect (Fig. 1a left and middle). The increase in the 
alpha-EEG became more prominent in the later period of sound presentation (whole 400-sec period: $p=.17$, latter 200-sec half: $p=.047$, final 100-sec period: $p=.021$ ). This is consistent with our previous study in which the hypersonic effect emerged after considerable delay (Oohashi et al., 2000).

When sound was presented exclusively to the ears through earphones (6 males and 9 females, 25-65 years of age), no difference between FRS and LFC alone in the alpha-EEG was observed (Fig. $1 \mathrm{~b}$ left and middle; whole 400 -sec period: $\mathrm{p}=.45$, latter 200-sec half: $p=.88$, final 100-sec period: $p=.41$ ). In contrast, when LFC was presented through earphones and HFC was presented through speakers to the front body surface of the subjects ( 7 males and 8 females, 25-65 years of age), the alpha-EEG power was significantly greater during FRS than during LFC alone in the later period of sound presentation (Fig. 1c left and middle; whole 400-sec period: $p=.054$, latter 200-sec half: $p=.029$, final 100-sec period: $p=.0020$ ). On the other hand, when the body surface of the subjects was insulated from exposure to HFC presented through speakers with a sound-insulating full-face helmet and a sound-insulating entire-body coat (5 males and 8 females, 25-65 years of age), the increase in the alpha-EEG during FRS was markedly suppressed (Fig. 1d left and middle; whole 400-sec period: $p=.42$, latter half of 200 -sec period: $p=.64$, final 100-sec period: $p=.47$ ). These data indicate that the hypersonic effect was evoked only when HFC was presented to the head and/or body surface.

Behavioral experiment. Behavioral measurement of CLL was consistent with the results of the EEG experiments. When both LFC and HFC were presented through speakers to subjects (5 males and 5 females, 25-65 years of age) (Fig. 1a right), or when LFC was presented through the earphones and HFC was presented through the speakers to subjects 
(5 males and 5 females, 31-65 years of age) (Fig. 1c right), the subjects spontaneously adjusted the sound to a significantly greater magnitude for more comfortable listening during presentation of FRS than during presentation of LFC alone. In contrast, subjects adjusted the listening level to similar magnitudes during presentation of FRS and LFC alone when both LFC and HFC were presented through earphones (3 males and 6 females, 25-50 years of age) (Fig. $1 \mathrm{~b}$ right, $p=.96$ ). When LFC and HFC were presented through earphones and speakers, respectively, with the body surface of subjects insulated from exposure to HFC (4 males and 5 females, $34-65$ years of age), the increase in the CLL during FRS was markedly suppressed (Fig. 1d right, $p=.27$ ).

\section{Discussion}

As the first step in exploring the biological mechanism of the hypersonic effect, we considered whether there was a possibility of any biological system other than the air-conducting auditory system involved in the emergence of the hypersonic effect. We tested for the emergence of the hypersonic effect under various sound presentation conditions in which the LFC and HFC were presented over the entire body surface through speakers or selectively into the ears through earphones. Two independent measurements based on differing principles were employed for this particular experiment, namely the physiological measurement of the alpha-EEG and the behavioral measurement of the CLL, and both measurements showed clear-cut results that we were not able to obtain in our pilot study using fMRI and PET. The results show that the measurement methods selected were appropriate for this research. 
Brain regions with neuronal activity that correlates with the power of the occipital alpha rhythm of an EEG can be categorized into three different types (Salek-Haddadi et al., 2003): (1) the cerebral cortex which serves as a generator of the alpha rhythm, such as the occipital cortex whose activity shows a negative correlation with the alpha rhythm; (2) the regions forming the cortico-subcortical circuit but not directly generating the alpha rhythm recorded on the scalp, such as the thalamus; and, (3) other regions indirectly and functionally affecting the appearance of the alpha rhythm, such as the limbic system and the brainstem. Type (2) and (3) are considered to be related to the changes in the alpha-EEG observed in the present study. When we reanalyzed the PET measurement of rCBF and the simultaneously recorded spontaneous EEG that we had previously reported (Oohashi et al., 2000) using principal component analysis (Nakamura et al., 2004), the first principal component, which corresponded to a set of brain regions showing the most prominent difference in rCBF between FRS and LFC alone, included the upper brainstem (midbrain), hypothalamus, thalamus, precuneus, prefrontal cortex, and anterior cingulate gyrus. Correlation analysis between this first principal component and the simultaneously recorded EEGs revealed that the alpha 2 component recorded from 7 electrodes in the central and parieto-occipital regions $(\mathrm{C} 3, \mathrm{C} 4, \mathrm{~T} 5, \mathrm{Pz}$, $\mathrm{T} 6, \mathrm{O} 1$, and $\mathrm{O} 2$ ) and the averaged alpha 2 potential across these electrodes correlated significantly with the conditional profile of the first component (Nakamura et al., 2004). This finding is compatible with our previous report (Sadato et al., 1998, Oohashi et al., 2000) and that of another group (Goldman et al., 2002). Furthermore, the finding compares well with our previous report that the activation of the brainstem and thalamus serve as a neurophysiological basis of the hypersonic effect. It is reasonable to consider, 
therefore, that the hypersonic effect detected by the increase in the power of the alpha-EEG in the present study reflects the activation of the deep-lying brain structure, including the brainstem and thalamus. These brain areas, containing distinct neuronal groups that are the major source of the monoaminergic projections to various parts of the brain (Role and Kelly, 1991), introduce approaching behaviors and are considered to be intimately connected with registering pleasurable sensations (Thompson, 1988).

CLL was used as a measure of the perception of subtle differences in sound quality that may not be consciously recognizable or may not otherwise be easily expressed by the subjects (Cullari and Semanchick, 1989, Namba and Kuwano, 1998). The basic strategy of this measurement is that subjects tend to receive preferable stimulus at a greater magnitude. It is conceivable, therefore, that the increased or decreased CLL observed in the present study may reflect change in approaching behaviors introduced by the activation or deactivation of the reward-generating system situated in the deep-lying brain structure. This explanation dovetails well with the results of the EEG experiment. Alternatively, the HFC might have a certain effect of suppression that could be observed only when the "suppressee," i.e., the LFC, is simultaneously presented, and the participants might raise the listening level to compensate for the concomitant suppression. The present experiment did not obviate this possibility.

The point of the present experimental design is to focus on the fact that the hypersonic effect does not emerge at the presentation of HFC alone but it emerges only when HFC and LFC were simultaneously presented. Therefore, we compared the listeners' responses under two experimental conditions: FRS and LFC alone in an experimental setting that clearly showed whether the air-conducting auditory system or 
another biological system was involved. The subjects did not perceive HFC alone as a sound when it was presented either through speakers or earphones. Nevertheless, when both the LFC and HFC were simultaneously presented through speakers not only to the conventional air-conducting auditory system but also to the entire body surface, which might have another sensing system, the power of the spontaneous EEG activity of the alpha 2 range was significantly enhanced and the subjects spontaneously adjusted the sound to a significantly greater magnitude for more comfortable listening than when LFC alone was presented through the speakers, indicating the emergence of the hypersonic effect in the given experimental setting. These results are in complete agreement with our previous reports (Oohashi et al., 2000, Oohashi et al., 1991, Oohashi et al., 2001, Yagi et al., 2003a, Yagi et al., 2002, Yagi et al., 2003b).

More importantly, the emergence of the hypersonic effect was not observed in any of the two indexes when both the LFC and HFC were presented selectively to the air-conducting auditory system through the earphones. Since a setting in which both LFC and HFC are presented selectively to the air-conducting auditory system without disturbance is ideal, we would expect to observe the hypersonic effect if it could be induced by the air-conducting auditory system alone. However, the fact that absolutely no hypersonic effect was observed under this condition demonstrates that the air-conducting auditory system does not respond to HFC.

By contrast, when LFC was presented selectively to the air-conducting auditory system through earphones and HFC was presented to the entire body surface through speakers, the hypersonic effect was observed with statistical significance in the indexes of both alpha-EEG and CLL. Since the earphones used in this experiment had a 2-to-3 
$\mathrm{mm}$ thick, injection-molded hard plastic portion inserted into the ear canal that completely prevented the HFC presented through the speakers from leaking into the ear canal, it is unlikely that the HFC reached the air-conducting auditory system. In addition, since the HFC was conducted through the air and the LFC through the ear canal, it is unlikely that the HFC and the LFC would cause a latent modulation in the air or in the ear canal or that the "modulated" LFC would induce the hypersonic effect through the auditory system. These findings suggest that (1) the presentation of HFC to the air-conducting auditory system is not necessary for the emergence of the hypersonic effect and (2) its presentation to the entire body surface other than ears can bring about the hypersonic effect.

In another experiment, the emergence of the hypersonic effect was markedly suppressed when LFC was presented to the ear through earphones and HFC was presented through speakers with sound insulators placed just in front of the subjects. This finding suggests that the HFC must directly reach the entire body surface, including the head, for the hypersonic effect to appear. We also noticed that when a small quantity of HFC, though greatly reduced, still reached the body surface of the subjects, the alpha-EEG and the CLL showed a non-significant trend toward an increase, suggesting that the degree of the emergence of the hypersonic effect may correlate with the amount of HFC reaching the body surface. This is consistent with our previous report that the HFC was transduced in a graded manner depending on the power of the HFC and the hypersonic effect increased in magnitude with an increase in power of the HFC (Yagi et al., 2003a). 
We can find no evidence so far to support a model with the conventional air-conducting auditory system alone involved in the emergence of the hypersonic effect, which is induced by non-stationary sounds containing rich HFC above the human audible range. The experimental findings in this study cannot be explained by the air-conducting auditory system alone; they can be explained with less contradiction by assuming the existence of some hitherto unknown sensing mechanism somewhere on the body surface, even the head. Pursuing the biological mechanism of the hypersonic effect only within the range of the previously recognized air-conducting auditory system not only presents serious limitations but also overlooks important findings. We must also consider the possible existence of an unrecognized sensing mechanism.

Although identification of the specific mechanism that participates in sensing and transducing HFC is beyond the scope of the present study, in future studies, research must include an examination of various sensory neuronal systems. For example, the somatosensory system conveys information about mechanical vibration of the body surface. Meissner corpuscles serve as sensors of vibratory stimuli with large amplitude and low frequency; Pacinian corpuscles as sensors of small amplitude and high frequency. Although the frequency response range of Meissner and Pacinian corpuscles is known to be $5-80$ and $80-600 \mathrm{~Hz}$ with a peak response at 30 and $200-400 \mathrm{~Hz}$, respectively (Douglas et al., 1978), these known mechanoreceptors may possess some unknown responsiveness to air vibration above the upper limit of the human audible range (i.e., above $20 \mathrm{kHz}$ ). Another example comes from a recent report on ultrasonic sound hearing (Lenhardt et al., 1991). People with normal hearing as well as those whose hearing is totally impaired due to a disturbance of the inner ear can successfully recognize the vibratory 
stimulus of ultrasound modulated by the human voice. Such a bone-conducting auditory system may well have some effect on the emergence of the hypersonic effect.

A recent study reported that general, non-sensory cells other than the receptors of the sensory nervous system have mechanosensitive channels and a cellular responsiveness to a wide range of mechanical stimuli from outside (Alenghat and Ingber, 2002). Mechanotransduction has drawn considerable attention as one of the fundamental functions of life phenomena in general. Sokabe and his colleagues (Kanzaki et al., 1999) have successfully identified the gene that encodes "a stretch-activated nonselective cation channel" in eukaryote. We should pay special attention to the possibility that, through such a mechanotransduction channel, HFC air vibrations might effect changes in a somatic cellular state, including intracellular messengers, genetic expression and control, metabolic regulation, enzymatic responses, membrane permeability and molecular diffusion. Such information might be conveyed via neurons or a non-neuronal system, including the hormonal and/or immune systems. This is not inconsistent with the idea that the effects of ultrasound as practically applied in the medical field are mediated by several non-neuronal mechanisms (Abbott, 1999), such as thermal changes, cavitation, micro-streaming, and physical and chemical consequences induced by the small displacement of microparticles in the body.

In conclusion, our study suggests that the emergence of the hypersonic effect can be observed only when some unknown information channel, not the air-conducting auditory nervous system, is activated. In future studies we must not ignore the possibility of the participation of one or more other unidentified sensing mechanisms. Our results support our previously proposed model, the two-dimensional sound 
perception model, in which inaudible HFC modulate brain functions by activating deep-lying brain structures via some non-auditory pathways when presented concurrently with audible LFC, thus evoking the hypersonic effect.

\section{Experimental Procedure}

Subjects. Healthy Japanese adult volunteers participated in the EEG and behavioral experiments. Data on the number, gender and age of the subjects that participated in each experiment are provided in the Results section. None of the subjects had any history of neurological or psychiatric disorders. Written informed consent was obtained from each of them before the experiment. The experiments were performed in accordance with the approval of the Ethics Committee, National Institute for Physiological Sciences. All the subjects had more than 5 years exposure to the actual HFC-rich sounds of the musical instruments used as a sound source.

Sound materials and presentation system. The sound stimulus was a traditional gamelan composition, "Gambang Kuta," of Bali Island, Indonesia, which contains a wealth of high frequencies with a conspicuously fluctuating structure and has been proven to induce the hypersonic effect. A bi-channel sound presentation system (Oohashi et al., 2000, Yagi et al., 2002) was used to present the sound stimulus (Fig. 2). Using high-pass and low-pass filters (CF-6FH and CF-6FL; NF Electronic Instruments, Yokohama, Japan) with crossover frequency of $22 \mathrm{kHz}$, cut-off attenuation of 80 $\mathrm{dB} /$ octave and ripple in the passband frequency of $\pm 1 \mathrm{~dB}$, we divided the source signals into audible LFC and inaudible HFC, and amplified them independently of each other. These signals were presented simultaneously or separately though speaker or earphone. 
In most audio systems conventionally used to present sound for determining sound quality, sounds containing HFC are presented as unfiltered source signals through an all-pass circuit, whereas sounds without HFC are produced by passing the source signals through a low-pass filter (Muraoka et al., 1978, Plenge et al., 1979). Thus, audible LFC are presented through different pathways that might have different transmission characteristics, including frequency response and group delay. In addition, inter-modulation distortion might differentially affect LFC. Therefore, any observed differences between the two sounds, those with and without HFC, might well result from differences in the audible LFC presented through the different pathways rather than from the existence of HFC. The bi-channel sound presentation system that we developed for a series of the studies on the hypersonic effect fundamentally overcomes such problems by presenting LFC through an identical pathway in the two sounds, those with and without HFC. Exact specifications of the sound source and the bi-channel sound presentation system (Authentic Signal Disc ARHS9002 and Authentic Hypersonic Sound System, Action Research Co., Ltd., Tokyo, Japan) have been described elsewhere (Yagi et al., 2002).

The speaker components of the system were placed approximately 2.0 meters from the subjects' ears. Subjects used custom-made closed-air type insert earphones without ear pads. The portion inserted into the ear canal was made of injection-molded hard plastic 2-to-3 mm thick. Both the right and left earphones contained two vibratory devices, one for LFC and the other for HFC. Fig. 3 shows the power spectra of the actual air vibration reproduced by the bi-channel sound presentation system and recorded 
with a microphone at the subject's position (4135, Brüel \& Kjær, Nærum, Denmark). The averaged power spectra of the entire 200 -sec piece of music used in the experiments were measured on a Fast Fourier Transform (FFT) analyzer (CF-5220, Ono Sokki, Tokyo, Japan) with 2048 sampling points, sampling frequency of $128 \mathrm{kHz}$, Hanning window and averaging of approximately 25,000. The HFC alone was presented through speaker or earphone to each subject who, blind to the presentation, was asked to press a button whenever he or she could detect anything at all, not just auditory sensations, other than silence. None of the subjects could distinguish the presentation of HFC alone from silence. This is compatible with the notion that human beings cannot perceive elastic vibrations in the frequency range above $20 \mathrm{kHz}$ as sound (Snow, 1931, Wegel, 1922, Durrant and Lovrinc, 1977).

Each of the EEG and behavioral experiments consisted of four sub-experiments: (a) Both LFC and HFC presented through speakers; (b) Both LFC and HFC presented through earphones; (c) LFC presented through earphones; HFC presented through speakers; (d) LFC presented through earphones; HFC presented through speakers but with sound insulators preventing exposure of the subject's head and body surface to HFC. In all experiments, two conditions were compared FRS (simultaneous presentation of LFC and HFC) and LFC alone. The order of the sub-experiments was randomized and counterbalanced across the subjects. All experiments were performed in an acoustically isolated room. Special attention was given to the subjects' immediate environment to avoid discomfort.

Measurement of EEG. In each experiment, there were two trials each for FRS and LFC alone with an inter-trial interval of several minutes in an A-B-B-A fashion, in which FRS 
and LFC alone were assigned to A and $\mathrm{B}$, or B and A, respectively, in a counterbalanced manner across subjects. The presentation of the sound in each trial lasted $400 \mathrm{sec}$, consisting of one repetition of the entire piece of music. Subjects were asked to keep their eyes naturally open. EEGs were recorded using a telemetric system (WEE-6112, Nihon-Koden, Tokyo, Japan) from 12 scalp sites (Fp1, Fp2, F7, Fz, F8, C3, C4, T5, Pz, T6, O1 and O2) according to the International 10-20 System using linked earlobe electrodes as a reference with a filter setting of $1-60 \mathrm{~Hz}(-3 \mathrm{~dB})$. The power spectrum of the EEG at each electrode was calculated using FFT analysis for every 2-sec epoch with an overlap of $1 \mathrm{sec}$, with a sampling frequency of $256 \mathrm{~Hz}$. The square root of the averaged power level in a frequency range of $10.0-13.0 \mathrm{~Hz}$ at each electrode position was calculated as the equivalent potential of EEGs in the alpha 2 band. To eliminate inter-subject variability, the data were normalized with respect to the mean value across all time epochs and conditions for each subject. After excluding epochs contaminated by artifacts, the data obtained from 7 electrodes in the centro-parieto-occipital region (C3, $\mathrm{C} 4, \mathrm{~T} 5, \mathrm{Pz}, \mathrm{T} 6, \mathrm{O} 1, \mathrm{O} 2)$ were averaged across all the analysis epochs (alpha-EEG) and compared between the two conditions: FRS and LFC alone. This EEG index has been shown to correlate significantly with the net activity of the neuronal network in the deep-lying brain structure including the brainstem, thalamus and hypothalamus, which are considered to be neuronal substrates of the hypersonic effect (Nakamura et al., 2004). Analysis of the whole alpha range $(8-13 \mathrm{~Hz})$ gave rise to the same results as that of the alpha2 band (10-13 Hz).

There was a considerable delay from the onset of sound presentation before a significant change in the EEG was observed (Oohashi et al., 2000), thus statistical 
evaluation (paired Student's t-test between FRS and LFC alone) was performed for the latter 200 -sec half and the last $100-\mathrm{sec}$ period as well as for the entire 400 -sec period of the sound stimulus. The scalp distribution of the change in the alpha 2 component between FRS and LFC alone was also evaluated by constructing colored contour line maps using 2,565 scalp grid points with linear interpolation and extrapolation (Duffy et al., 1979, Ueno and Matsuoka, 1976) based on z-values calculated from pair-wise comparisons of the strength of the alpha 2 component at each electrode.

Measurement of CLL. The same four sub-experiments were performed for CLL measurement as we did in the EEG experiments. In each experiment, two sessions each for FRS and LFC alone were performed with an inter-session interval of several minnutes in a counterbalanced manner across subjects. The experiment was performed under a double blind condition, namely one experimenter controlled the presentation of the experimental sound and another experimenter who did not know the experimental condition gave the instruction to the subjects and measured the listening level. The subjects were also blind as to the conditions of the sessions. One session consisted of 5 trials, each of which was a $200-\mathrm{sec}$ presentation of the same sound stimulus. Throughout a single session, either FRS or LFC alone was presented. The listening level was measured as equivalent continuous A-weighted sound pressure level (LAeq) using an integrated sound level meter (LA-5111; ONO SOKKI, Yokohama, Japan). Note that a measured sound pressure level is not affected by the existence of HFC above $22 \mathrm{kHz}$ because only the $L$ Aeq of sounds below $20 \mathrm{kHz}$ was measured in this method. In fact, FRS and LFC alone showed equal levels of $L$ Aeq within $\pm 0.1 \mathrm{~dB}$ at the same volume in the present experimental setting. In the first trial, subjects listened to the 
sound stimulus at a fixed level adjusted to $79.5 \mathrm{~dB} L$ Aeq at the listening position when the sound stimulus was presented through speakers, and at a subjectively equivalent listening level when it was presented through earphones. During the next three trials, subjects were requested to freely adjust the listening level to what they considered to be comfortable using a remote controller with an up-down switch that controlled the motorized fader (PGFM3000; Penny \& Giles, Gwent, UK) positioned between the player and the pre-amplifier. No visual or tactile information on the volume was given to subjects when they adjusted the listening level. Then, during the final trial, each subject listened to the sound fixed at the level that they had selected at the end of the preceding trial. The level measured in the final listening trial and adjusted in terms of $L$ Aeq was considered to be the CLL. Statistical evaluation was made using paired Student's t-test between FRS and LFC alone. 


\section{Acknowledgements}

We thank Dr. Yoshio Yamasaki, Waseda University, for the use of his developed signal processing system, the Yamashiro Institute of Science and Culture for valuable technical support, and Dr. Rieko Osu, ATR Computational Neuroscience Laboratories for her valuable comments on the manuscript. This work was supported in part by the Ministry of Education, Culture, Sports, Science and Technology of Japan, through the Grants-in-aid for Scientific Research and for the Creation of Innovations through Business-Academic-Public Sector Cooperation, and the Japan Science and Technology Agency. 


\section{References}

ABBOTT, J. G. (1999) Rationale and derivation of MI and TI--a review. Ultrasound Med Biol, 25, 431-41.

ALENGHAT, F. J. \& INGBER, D. E. (2002) Mechanotransduction: all signals point to cytoskeleton, matrix, and integrins. Sci STKE, 2002, PE6.

CULLARI, S. \& SEMANCHICK, O. (1989) Music preferences and perception of loudness. Percept. Mot. Skills., 68, 186.

DOUGLAS, P. R., FERRINGTON, D. G. \& ROWE, M. (1978) Coding of information about tactile stimuli by neurones of the cuneate nucleus. $J$ Physiol, 285, 493-513.

DUFFY, F. H., BURCHFIEL, J. L. \& LOMBROSO, C. T. (1979) Brain electrical activity mapping (BEAM): a method for extending the clinical utility of EEG and evoked potential data. Ann. Neurol., 5, 309-321.

DURRANT, J. D. \& LOVRINC, J. H. (1977) Bases of hearing science, Hagerstown, Lippincott Williams \& Wilkins.

GOLDMAN, R. I., STERN, J. M., ENGEL, J., JR. \& COHEN, M. S. (2002) Simultaneous EEG and fMRI of the alpha rhythm. Neuroreport, 13, 2487-92.

KANZAKI, M., NAGASAWA, M., KOJIMA, I., SATO, C., NARUSE, K., SOKABE, M. \& IIDA, H. (1999) Molecular identification of a eukaryotic, stretch-activated nonselective cation channel. Science, 285, 882-6.

LENHARDT, M. L., SKELLETT, R., WANG, P. \& CLARKE, A. M. (1991) Human ultrasonic speech perception. Science, 253, 82-5.

MURAOKA, T., YAMADA, Y. \& YAMAZAKI, M. (1978) Sampling-frequency considerations in digital audio. J. Audio. Engineer. Soc., 26, 252-256. 
NAKAMURA, S., HONDA, M., MORIMOTO, M., YAGI, R., NISHINA, E., KAWAI, N., MAEKAWA, T. \& OOHASHI, T. (2004) Electroencephalographic evaluation of the hypersonic effect. Society for Neuroscience Abstract, 752.14.

NAMBA, S. \& KUWANO, S. (1998) Method of Psychological Measurement for Hearing Research (in Japanese), Tokyo, Colona Publishing.

OOHASHI, T., NISHINA, E. \& HONDA, M. (2001) Multidisciplinary study on the hypersonic effect. IN SHIBASAKI, H., FUKUYAMA, H., NAGAMINE, T. \& MIMA, T. (Eds.) Inter-areal coupling of human brain function. Amsterdam, Elsevier Science.

OOHASHI, T., NISHINA, E., HONDA, M., YONEKURA, Y., FUWAMOTO, Y., KAWAI, N., MAEKAWA, T., NAKAMURA, S., FUKUYAMA, H. \& SHIBASAKI, H. (2000) Inaudible high-frequency sounds affect brain activity: hypersonic effect. J. Neurophysiol., 83, 3548-58.

OOHASHI, T., NISHINA, E., KAWAI, N., FUWAMOTO, Y. \& IMAI, H. (1991) High-frequency sound above the audible range affects brain electric activity and sound perception. Proceedings of 91st Audio Engineering Society convention. New York, Audio Engineering Society.

PLENGE, G., H., JAKUBOWSKI, H. \& SCHONE, P. (1979) Which bandwidth is necessary for optimal sound transmission. Proceedings of 62nd Audio Engineering Society convention. Brussels, Audio Engineering Society.

ROLE, L. W. \& KELLY, J. P. (1991) The brain stem: Cranial nerve nuclei and the monoaminergic systems. IN KANDEL, E. R., SCHWARTZ, J. H. \& JESSELL, T. M. (Eds.) Principle of Neural Science. Connecticut, Appleton \& Lange. 
SADATO, N., NAKAMURA, S., OOHASHI, T., NISHINA, E., FUWAMOTO, Y., WAKI, A. \& YONEKURA, Y. (1998) Neural networks for generation and suppression of alpha rhythm: a PET study. Neuroreport, 9, 893-7.

SALEK-HADDADI, A., FRISTON, K. J., LEMIEUX, L. \& FISH, D. R. (2003) Studying spontaneous EEG activity with fMRI. Brain Res Brain Res Rev, 43, 110-33.

SNOW, W. B. (1931) Audible frequency ranges of music, speech and noise. J. Acoust. Soc. Am., 3, 155-166.

THOMPSON, J. G. (1988) The psychobiology of emotions, New York, Plenum Press.

UENO, S. \& MATSUOKA, S. (1976) Topographic display of slow wave types of EEG abnormality in patients with brain lesions. Iyoudenshi To Seitai Kogaku, 14, $118-124$.

WEGEL, R. L. (1922) The physical examination of hearing and binaural aids for deaf. Proc. Natl. Acad. Sci. U. S. A., 8, 155-160.

YAGI, R., NISHINA, E., HONDA, M. \& OOHASHI, T. (2003a) Modulatory effect of inaudible high-frequency sounds on human acoustic perception. Neurosci Lett, $351,191-5$.

YAGI, R., NISHINA, E., KAWAI, N., HONDA, M., MAEKAWA, T., NAKAMURA, S., MORIMOTO, M., SANADA, K., TOYOSHIMA, M. \& OOHASHI, T. (2002) Auditory Display for Deep Brain Activation: Hypersonic Effect. The 8th International Conference on Auditory Display. Kyoto.

YAGI, R., NISHINA, E. \& OOHASHI, T. (2003b) A method for behavioral evaluation of the "hypersonic effect". Acoust Sci \& Tech, 24, 197-200. 


\section{Figure legends}

Figure 1. Electroencephalographic activity and listening level adjusted by the subjects during different experimental conditions. (a) Both LFC and HFC presented through speakers. (b) Both LFC and HFC presented through earphones. (c) LFC presented through earphones; HFC presented through speakers. (d) LFC presented through earphones; HFC presented through speakers but with sound insulators preventing exposure of the subject's body surface to HFC. (Left panel) Mean $(+\mathrm{SE})$ value of the normalized spontaneous alpha-EEG across all subjects. (Middle panel) The maps showing the scalp distribution of $z$-values calculated from pair-wise comparisons of the strength of the alpha 2 component at each electrode during the last 100 -sec period. Darker red represents a greater power during presentation of FRS than during presentation of LFC alone. (Right panel) Transition of averaged listening levels across subjects. The bars show the mean difference $(+\mathrm{SE})$ within subjects between comfortable listening level (CLL) during presentation of FRS and that during presentation of LFC alone. Positive values indicate a higher CLL during FRS.

Figure 2. The experimental setup employed for this study. Using programmable high-pass and low-pass filters with a crossover frequency of $22 \mathrm{kHz}$ and a cut-off attenuation of $80 \mathrm{~dB} /$ octave, each of the source stereo signals was divided into LFC and HFC, and the two signals were independently amplified. They were presented either separately or simultaneously through speakers or earphones.

Figure 3. Averaged power spectra of the various sound materials calculated for the entire period of the sound presentation. Data of the left channel are shown. (a) The 
electric signal of the sound source. (b) The sounds reproduced by the bi-channel sound presentation system through the speaker. Power was calculated based on the data recorded at the subject's position. (c) Attenuation of HFC through the speaker by clothes and sound insulators used. Only HFC was presented through speakers and the sound was recorded at the subject's position behind each sound insulator. Subjects wore T-shirts during all experiments except when they wore a sound-insulating entire-body coat. (d) The sounds reproduced by the bi-channel sound presentation system through the earphone. Power was calculated from a signal recorded $3.5 \mathrm{~cm}$ from the diaphragm, corresponding to the average length of an ear canal in adults. 

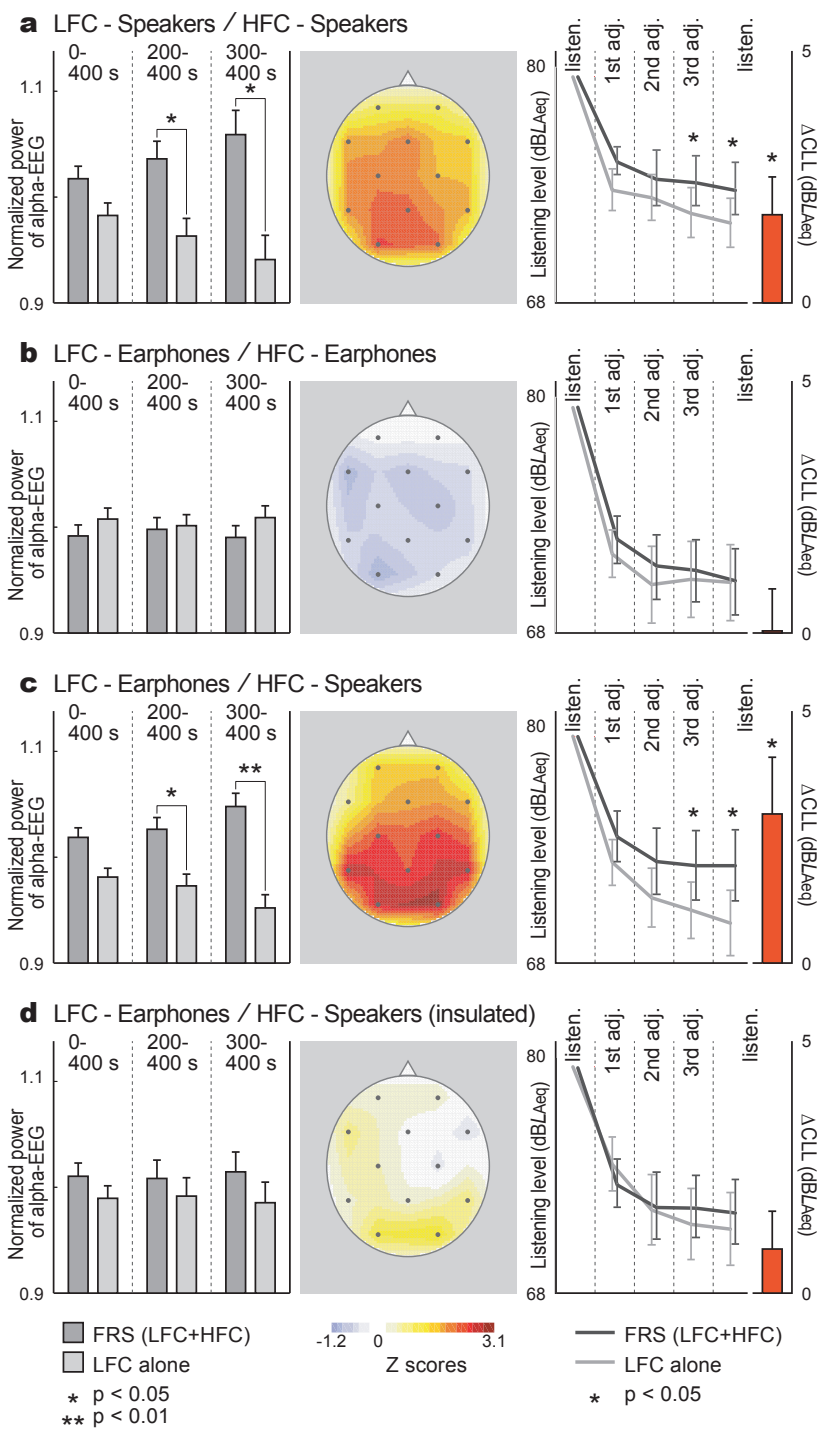

Figure 1 
Figure 2

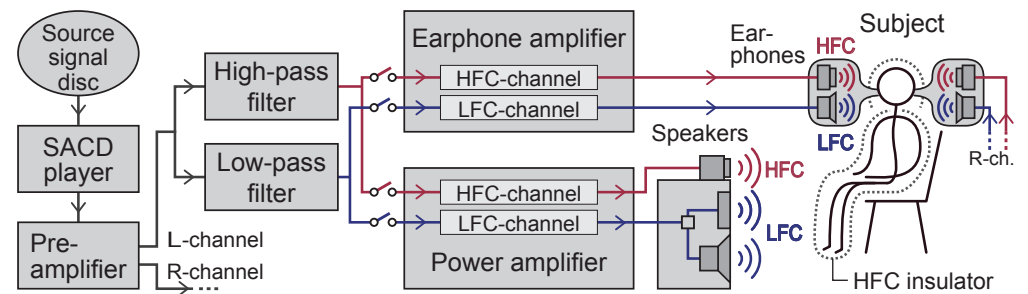

Figure 2 


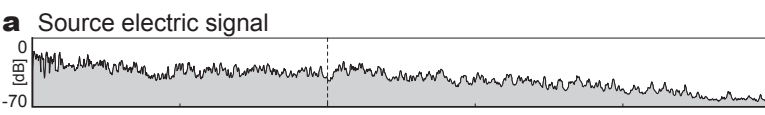

b Sounds through speaker system

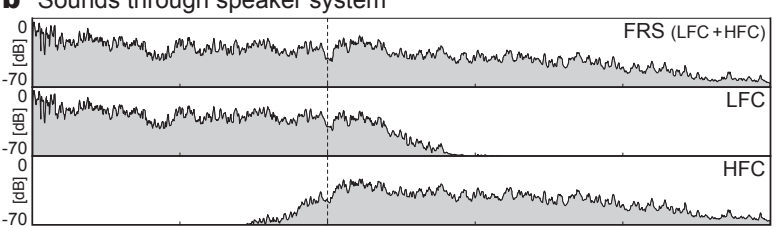

c Attenuated HFC through speaker system

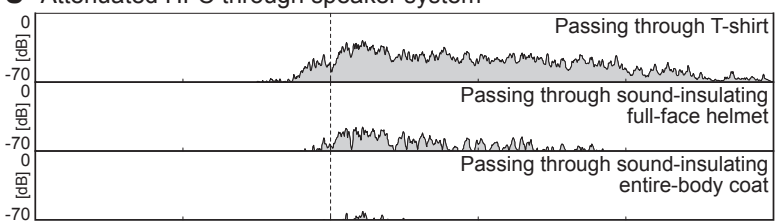

d Sounds through earphone system

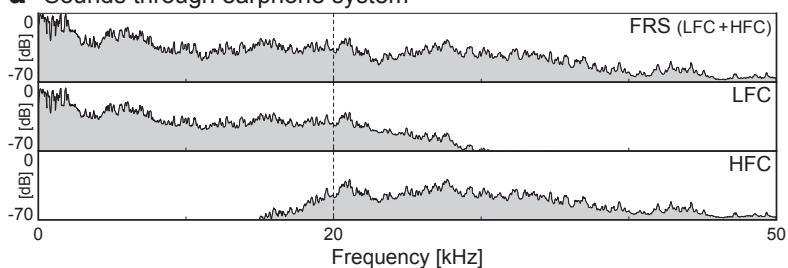

Figure 3 